\title{
IONIC CONDUCTIVITY OF STABILIZED ZIRCONIA NETWORKS IN COMPOSITE SOFC ELECTRODES
}

\author{
Keiji Yamahara $^{+}$, Tal Z. Sholklapper ${ }^{+}$ \\ Craig P. Jacobson ${ }^{+}$, Steven J. Visco ${ }^{+}$ \\ Lutgard C. De Jonghe ${ }^{*+} \#$ \\ ${ }^{+}$Materials Science Division, Lawrence Berkeley National Laboratory, \\ University of California, Berkeley, CA 94720, USA, \\ $\dagger$ Department of Physics \\ \# Department of Materials Science and Engineering \\ University of California at Berkeley \\ Berkeley CA 94720
}

\begin{abstract}
The effective oxygen conductivities in the zirconia networks of porous LSM-YSZ and LSM-SYSZ composites [i.e. $\mathrm{La}_{0.85} \mathrm{Sr}_{0.15} \mathrm{MnO}_{3}-\left(\mathrm{Y}_{2} \mathrm{O}_{3}\right)_{0.08}\left(\mathrm{ZrO}_{2}\right)_{0.92}$ and $\left.\mathrm{La}_{0.85} \mathrm{Sr}_{0.15} \mathrm{MnO}_{3}-\mathrm{c}_{2} \mathrm{O}_{3}\right)_{0.1}\left(\mathrm{Y}_{2} \mathrm{O}_{3}\right)_{0.01}\left(\mathrm{ZrO}_{2}\right)_{0.89}$, respectively] were evaluated by an $\mathrm{AC}$ impedance technique using specimens in which LSM was removed by hydrochloric acid leaching. The oxygen conductivities of porous YSZ and SYSZ alone followed a Koh-Fortini relationship. LSM-containing zirconia network conductivities were additionally decreased by the presence of the LSM, presumably by increased grain boundary resistances. Constriction resistances were estimated to have a minor effect.
\end{abstract}

PACS: Electrochemical energy conversion (84.60 D); Fuel cell (84.6 D)

Keywords: Composite cathode; LSM; zirconia network conductivity; solid oxide fuel cells; reduced temperature SOFCs.

\footnotetext{
"Corresponding author. Tel.: +1-510-486-6138; fax: +1-510-486-4881.

E-mail address: dejonghe@lbl.gov.
} 


\section{Introduction}

Lowering the operation temperature of solid oxide fuel cells (SOFCs) from $1000^{\circ} \mathrm{C}$ to $600-800^{\circ} \mathrm{C}$ may allow for a significant cost reduction for solid oxide fuel cell (SOFCs) systems by broadening the choice of compatible materials. At the same time, as the operating temperatures are decreased, the oxygen ion conductivities in typical cathode materials, such as lanthanum strontium maganates (LSM), is significantly reduced, adversely affecting the oxygen reduction reaction rate. Inclusion of a material with comparatively high oxygen-ion conductivity can improve the cathode performance significantly. The presence of the network of the YSZ electrolyte particles in the cathodes leads to an extended distribution of triple phase boundaries from the electrolyte /cathode interface into the cathode $[1,2]$ and effectively enhances the cathode's ion transport [1]. Attempts to utilize oxygen ion conductors such as doped ceria [3] and lanthanum strontium doped gallates [4] instead of YSZ as a component of composite cathodes have shown some success. However, it is not clear if the high ionic conductivities of these supporting electrolytes are dominant in the comparative enhancement of the cathode activity. One consideration is that the performance of composite cathodes strongly depends on the microstructure; another is that reactions between the supporting electrolytes and the cathode material can negate the benefits of the enhanced oxygen ion transport. Zirconia-based supporting electrolytes are attractive because of their compatibility with many perovskite electrode materials. In particular, scandia doped zirconia (SSZ), as a component of composite cathodes may be a good choice, since it possesses a higher oxygen-ion conductivity than YSZ. The authors [5] previously evaluated the performances SOFCs with composite LSM - SYSZ (yttria co- 
doped SSZ) cathodes and found the cathode performance indeed to be improved compared to LSM-YSZ composite cathodes. However, since potential differences in the composite cathode microstructures remained unclear, it was necessary to determine unambiguously the actual ionic conductivity of the supporting electrolyte network in the composite cathodes. In the present paper we report the evaluation of the oxygen conductivities of the YSZ or the SYSZ network component of the LSM-zirconia composite cathodes.

\section{Experimental}

LSM powder [i.e. $\mathrm{La}_{0.85} \mathrm{Sr}_{0.15} \mathrm{MnO}_{3}$ ] was synthesized by a glycine-nitrate method [6], and calcined at 1473K. YSZ [i.e. $\left(\mathrm{Y}_{2} \mathrm{O}_{3}\right)_{0.08}\left(\mathrm{ZrO}_{2}\right)_{0.92}$ ] and SYSZ [i.e. $\left.\left(\mathrm{Sc}_{2} \mathrm{O}_{3}\right)_{0.1}\left(\mathrm{Y}_{2} \mathrm{O}_{3}\right)_{0.01}\left(\mathrm{ZrO}_{2}\right)_{0.89}\right]$ powders were provided by the Tosoh Corporation and Daiichi Kigenso Kagakukogyo, respectively. The calcined LSM powder was mixed with YSZ or SYSZ in a weight ratio of 1:1, and attritor-milled together with Menhaden fish oil (MFO, 3 wto relative to ceramics) using zirconia milling balls, in iso-propanol (IPA), for $1 \mathrm{hr}$, at 550 rpm. After drying, the powder was ground with mortar and pestle, and passed trough a $150 \mu \mathrm{m}$ mesh sieve. Powder samples of 0.59 were then uniaxially pressed into pellets with diameter $1.25 \mathrm{~cm}$, at $11.6 \mathrm{MPa}$, using a stainless steel die. The resulting compacts were sintered at a temperature in the range of 1323 to $1773 \mathrm{~K}$, for $4 \mathrm{hrs}$, in air. Dilatometry (Orton model 1600D) characterized the densification of the powder pellets.

$$
\text { To measure the oxygen conductivity of the zirconia }
$$


network in the sintered composites, the sintered pellets were soaked in a concentrated hydrochloric acid solution for two days, at room temperature, to dissolve LSM. Subsequently, both sides of the pellets were sputter-coated with Au electrodes. A Pt-wire current collector was then affixed with Au paste. The conductivities of the pellets were measured by AC impedance, using a frequency response analyzer and a potentiostat (Solartron 1260 and 1286, respectively). AC impedances were measured between 623 and $1123 \mathrm{~K}$ in air, over a frequency range between 0.1-10 MHz, with an applied peak-to-peak voltage of $50 \mathrm{mV}$.

XRD measurements were also performed for the sintered specimens before and after the $\mathrm{HCl}$ etching using a diffractometer (Siemens D-500) with $\mathrm{CuK}_{\alpha}$ radiation in the range $2 \theta=10-80^{\circ}$.

Samples of SYSZ with 1 and 10 wt\% LSM were also
prepared in a manner similar to the one described above, and
their ionic conductivities at $1073 \mathrm{~K}\left(800^{\circ} \mathrm{C}\right)$ were measured by
AC impedance.

\section{Results and Discussion}

It can be expected that the effective oxygen-ion conductivities depend significantly on the porosity of the samples, and therefore, the densities of all samples was determined. The densities of the various samples after sintering are shown in Fig 1. As expected, the sintered densities increase with sintering temperatures. Fig. 2 compares the sintered densities of the composite samples with those of YSZ and SYSZ without LSM. It is evident that the presence of LSM significantly increased the densification of 
the LSM-YSZ composites, but had a comparatively lower effect on the LSM-SYSZ or actually impeded densification above $1473 \mathrm{~K}\left(1200^{\circ} \mathrm{C}\right)$. Fig. 3 shows the dilatometry traces of YSZ, LSM, and SYSZ compacts, after correction for thermal expansion. These data show that the SYSZ and LSM compacts had nearly identical densification behavior, while the YSZ densified more slowly. The effect of the LSM on the densification behavior of the composites may then, at least in part, be understood as resulting from the effective compressive stress that the faster-shrinking LSM network exerts on the YSZ network.

The densities of the samples after acid leaching shown in Fig 1 thus reflect the actual measured densities of the remaining zirconia networks.

The microstructure of samples sintered at $1423 \mathrm{~K}\left(1150^{\circ} \mathrm{C}\right)$ for 4 hours, are shown in Fig. 4. A striking difference between the porous YSZ network of the LSM-YSZ (Fig. 4c) and the porous YSZ (no LSM, Fig 4e) is the particulate nature and difference in uniformity of the networks. The significance of the contribution of the ligament constrictions to the resistance of the networks may be estimated by considering the constrictions as a sinusoidal perturbation with amplitude A of the surface of a cylinder of mean radius $\bar{r}$, as depicted in Fig. 5. This simple model ignores the detailed solution of the current flow, but may serve as a reasonable estimate of the effect. The consequence is that the resistance $\mathrm{R}$ becomes function of the relative amplitude of the perturbation, $\mathrm{k}=\mathrm{A} / \overline{\mathrm{r}}$. As a result, $\mathrm{R}$ becomes $\mathrm{R}(\mathrm{k})=\mathrm{R}(0) \mathrm{xY}(\mathrm{k})$, where

$$
Y(k)=\left[\int_{0}^{2 \pi}\left(1 /(1+k \cdot \sin x)^{2}\right) d x\right] \cdot\left[2 \pi / \int_{0}^{2 \pi}(1+k \cdot \sin x)^{2} d x\right]
$$

The first part of this expression relates to the resistance of the shape shown in Fig.5, 
while the second part corrects for volume changes with changing $\mathrm{k}$. The calculated values of $\mathrm{Y}(\mathrm{k})$ shown in Fig. 5 indicate that the inhomogeneity due to constriction resistance at particle contacts or, more generally, clustering do not become significant for perturbations that are less than $0.3-0.4$ of the mean column radius. The majority of the strings of particles in the networks fall within this range. It is therefore concluded that the detailed network geometry was not a dominant factor in differences between the measured resistances and those expected from the porosity effect alone.

Fig. 6 shows the oxygen conductivities of LSM-zirconia and pure zirconias. The oxygen ion conductivities in LSM-zirconias are about an order of magnitude lower than those in dense zirconia polycrystals (such as specimens sintered at $1673 \mathrm{~K}$ ), while having slightly stronger temperature dependences. The acid-leached composites all showed similar conductivities; a direct comparison is, however, not appropriate since the densities of the various samples differs for the same sintering treatment.

Acid leaching also affected the reference SYSZ and YSZ porous compacts (without LSM), reducing their ionic conductivity by about $30 \%$. The total conductivities of LSM-zirconia mixtures are shown in Fig. 7a and 7b, as a function of temperature before and after acid leaching. For both LSM-zirconia systems, the total conductivities decreased by more than two orders of magnitude after the etching, while the temperature dependence increases. This dramatic change in total conductivity results from the removal of the electronically conducting LSM network by the acid leaching process. X-ray diffraction (XRD) analysis indicated an increasing presence of lanthanum zirconate in both LSM-YSZ and LSM-SYSZ composites from about $1 \mathrm{vol} \%$ to as much as $10 \mathrm{vol} \%$ following sintering from 1373 to $1523 \mathrm{~K}\left(1250^{\circ} \mathrm{C}\right)$ respectively. However, no 
evidence of lanthanum zirconate or LSM could be detected after the acid leaching. It can therefore be concluded that an increasing fraction of the zirconia network was removed as lanthanum zirconate by the leaching process for samples sintered at higher temperatures, and that the oxygen-ion conductivities of the remaining zirconia-network were not directly affected by the lanthanum zirconium oxide formation.

It is interesting to note here that in spite of the evident differences in the zirconia network densities following sintering at the various temperatures, the YSZ and SYSZ zirconia networks had nearly identical oxygen ion conductivities, regardless of the zirconia composition or the density within the examined density range. This would indicate that the ionic conductivities of the zirconia supporting electrolyte network was dominated by some other factor.

Fig 8. plots the normalized conductivities at $1073 \mathrm{~K}\left(800^{\circ} \mathrm{C}\right)$ of the LSM-free YSZ and SYSZ samples sintered to different densities. The data have been fitted to the empirical Koh-Fortini [7]:

$$
\frac{\sigma_{0}}{\sigma(\xi)}=(1-\xi) /\left(1+11 \xi^{2}\right)
$$

relationship, where $\xi$ is the porosity, $\sigma(\xi)$ is the measured conductivity at porosity $\xi$, and $\sigma_{0}$ is the conductivity of the fully dense material. While the Koh-Fortini relationship was developed for porous metals, it fits the data of the LSM-free porous zirconias remarkably well. It should also be pointed out here that this relationship ignores possible contributions of resistive grain boundaries. The actual values of the conductivities of the fully dense YSZ and SYSZ at $1073 \mathrm{~K}$ were 0.0671 and $0.0236 \mathrm{Scm}^{-1}$, respectively. Also included in Fig 8 are the conductivities of the YSZ and SYSZ networks of the 
composites, normalized to the conductivity of the $0 \%$ porosity YSZ. It can be seen that the zirconia network conductivities of the composites fall consistently below the KohFortini (KF) relationship, although less evident at higher porosities. In addition, the conductivities of the SYSZ networks of the composites together with the conductivities of SYSZ samples containing 1 and $10 \mathrm{wt} \%$ of LSM, normalized to conductivity of the $0 \%$ porosity SYSZ are also included in this graph. The latter conductivities are also significantly below the KF relationship of the corresponding LSM-free samples, indicating that the effective oxygen ion conductivity has been decreased by the presence of the LSM. For the LSM- containing zirconia composites, it may then be proposed to construct a new KF-like dependence on porosity that is normalized to 0-porosity conductivity that has been lowered by the presence of LSM. Such a relationship is suggested in Fig 8. Within the network density range of this study, the decrease in oxygen ion conductivity is as much as a factor of 3 below that expected on the basis of porosity alone. The relative insensitivity of the YSZ and SYSZ network ionic conductivity of the composites to network density, in the range of densities accessible in this study, would indicate that, at fixed porosity, the dominant factor contributing to the difference between the effective oxygen ion conductivity expected on the basis of porosity alone and the actual one, is an increase in the grain boundary resistance of the particles constituting the zirconia supporting electrolyte network.

\section{Conclusion}

The oxygen conductivities in LSM-YSZ and LSM-SYSZ were evaluated by an AC impedance technique using specimens in which LSM was removed by an $\mathrm{HCl}$ acid 
leaching process. The effective oxygen ion conductivities of porous, LSM-free SYSZ and YSZ samples conductivities of followed a Koh-Fortini relationship, indicating the dominant effect of porosity on conductivity of these samples. The constriction resistance between the particles of the porous network was considered to be minor, based on a simple model evaluation.

Zirconia networks in LSM -zirconia composites had an effective oxygen ion conductivity that was significantly below that expected on the basis of average porosity alone. It was concluded that, after porosity, the dominant factor contributing to this lowered effective oxygen ion conductivity could most likely be attributed to an increase in the grain boundary resistance of the particles constituting the zirconia supporting electrolyte network as a result of their chemical modification due to contact with LSM.

\section{Acknowledgements}

This work was supported by the U.S. Department of Energy, National Energy Technology Laboratory. KY is grateful for support from the Mitsubishi Chemical Corporation. The authors also thank Daiichi Kigenso Kagakukogyo for supplying the SYSZ powder. 


\section{References}

1. H. Yokokawa, T. Horida, in High Temperature Solid Oxide Fuel Cells, S. C. Singhal and K. Kendall editors, p.136, Elsevier Advanced Technology, Oxford, UK (2003).

2. J. H. Choi, J. H. Jang, S. M. Oh, Electrochimica Acta, 46, 867 (2001).

3. T.J. Armstrong, A.V. Virkar AV, J. Electrochem. Soc. 149(12), A1565 (2002).

4. E.P. Murray,S.A. Barnett, in SOFC VI, S. C. Singhal and M. Dokiya, Eds., PV 9919, p. 369, The Electrochemical Society Proceedings Series, Pennington, NJ, (1999).

5. K. Yamahara, C.P Jacobson, S.J Visco, L.C. De Jonghe, Solid State Ionics, in press (2004).

6. L.A. Chick, L.R. Pederson, G.D. Maupin, J.L. Bates, L.E. Thomas, and G.J. Exarhos, Mater. Lett., 10 (1-2), 6 (1990).

7. J.C.Y. Koh and A. Fortini, Int. J. Heat Mass Transfer, 16 (1973) 2013. 


\section{Table and figure captions}

Fig. 1 Densities as a function of sintering temperature for LSM-YSZ and LSM-SYSZ composites, and for the corresponding YSZ and SYSZ networks following acid leaching.

Fig. 2 Densities as a function of sintering temperature for LSM-YSZ and LSM-SYSZ composites, and for LSM-free YS and SYSZ

Fig. 3. Densification traces for SYSZ, LSM, and YSZ, corrected for thermal expansion.

Fig. 4. Scanning electron microscope images of the fracture surfaces of various samples sintered at $1150^{\circ} \mathrm{C}$ showing the microstructures of the various samples.

Fig. 5. $\mathrm{Y}(\mathrm{k})$, showing the relative increase in resistivity of a sinusoidally perturbed column. A is the amplitude of the perturbation, while $\bar{r}$ is the mean radius.

Fig. 6. Effective oxygen-ion conductivity of SYSZ and YSZ (LSM-free) and of the SYSZ and YSZ network after acid leaching of the corresponding LSM-zirconia composites.

Fig 7. a. Total conductivity (electronic+ionic) as a function of temperature for the LSMYSZ composites and the corresponding YSZ networks, following sintering at the indicated temperatures.

b.Total conductivity (electronic+ionic) as a function of temperature for the LSMSYSZ composites and the corresponding SYSZ networks, following sintering at the indicated temperatures. The dotted lines in Figs. a and $\mathrm{b}$ are identical, illustrating the close values for both the YSZ and the SYSZ networks of the composites.

Fig. 8. Normalized effective ionic conductivities. The LSM-free samples follow a KohFortini relationship. The samples containing LSM have a reduced relative conductivity. 


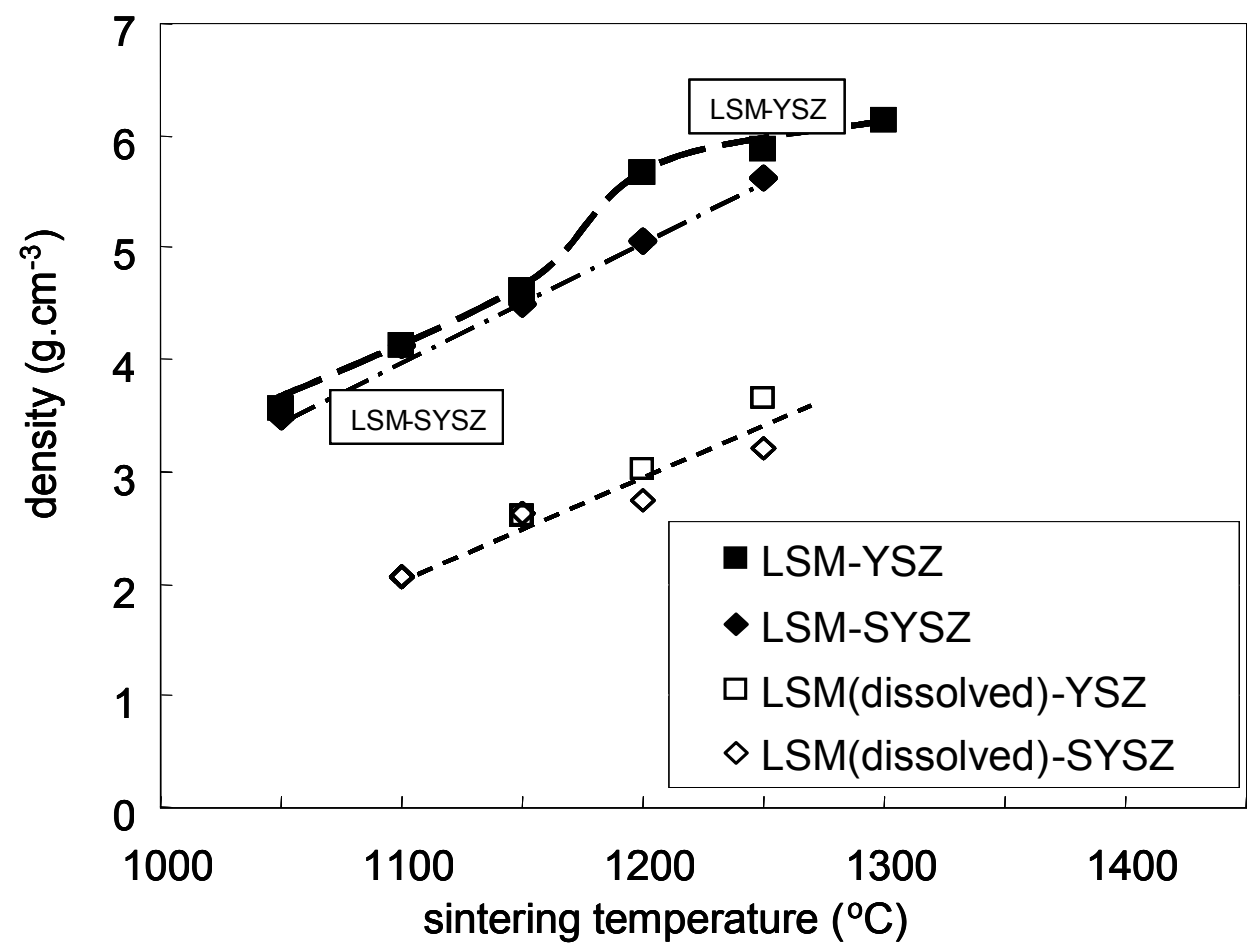

Fig.1 


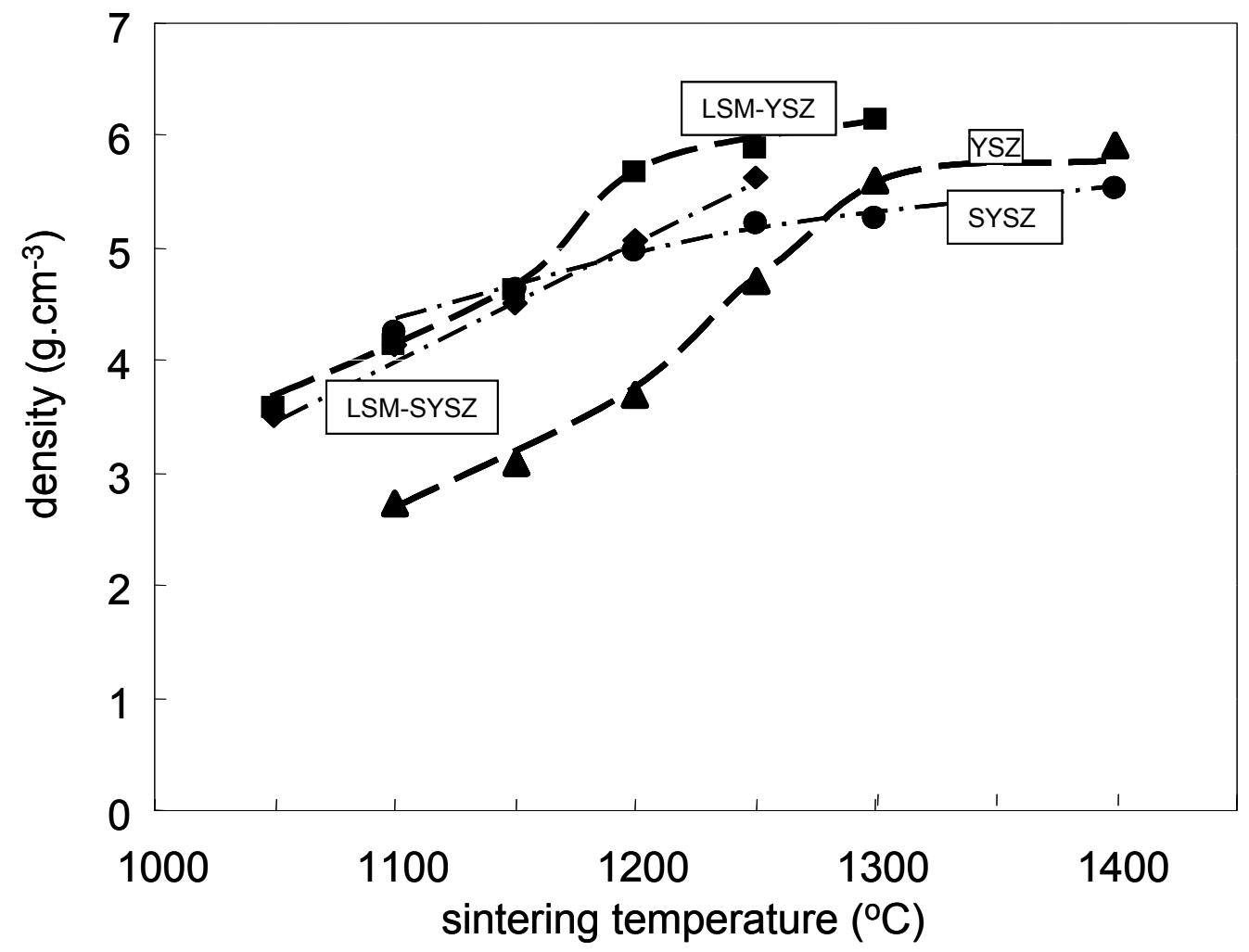

Fig. 2 


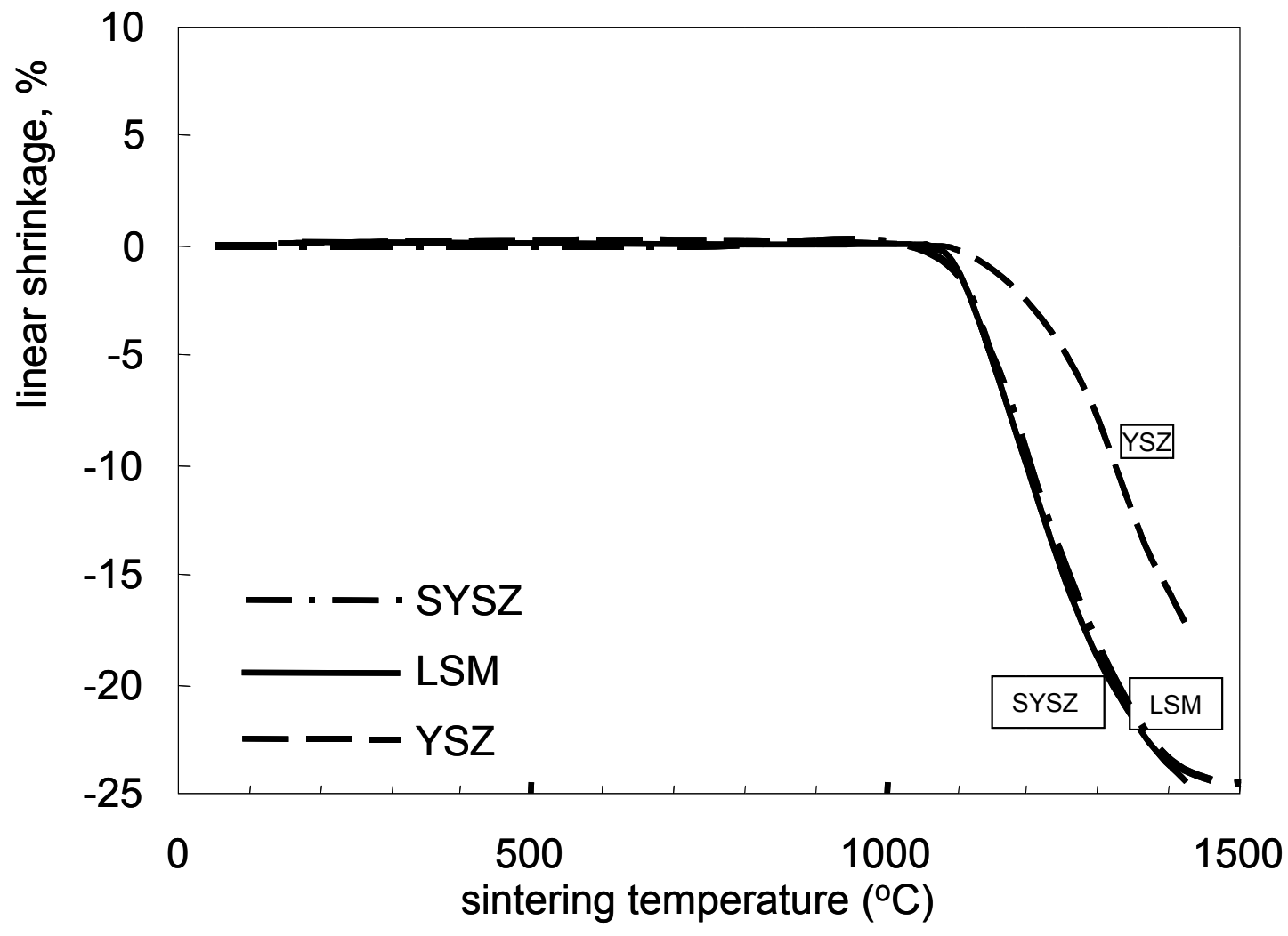

Fig. 3 


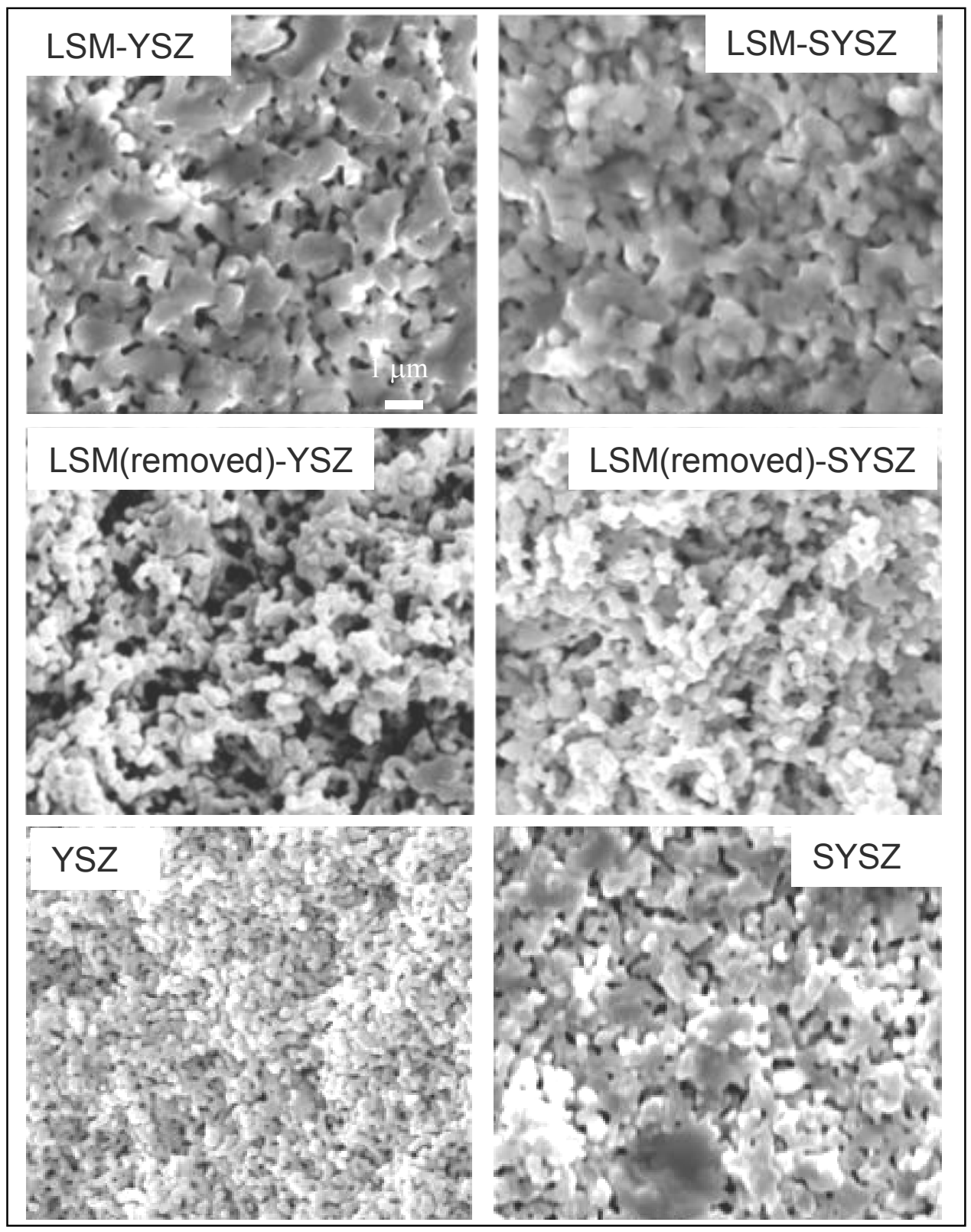

Fig.4. 


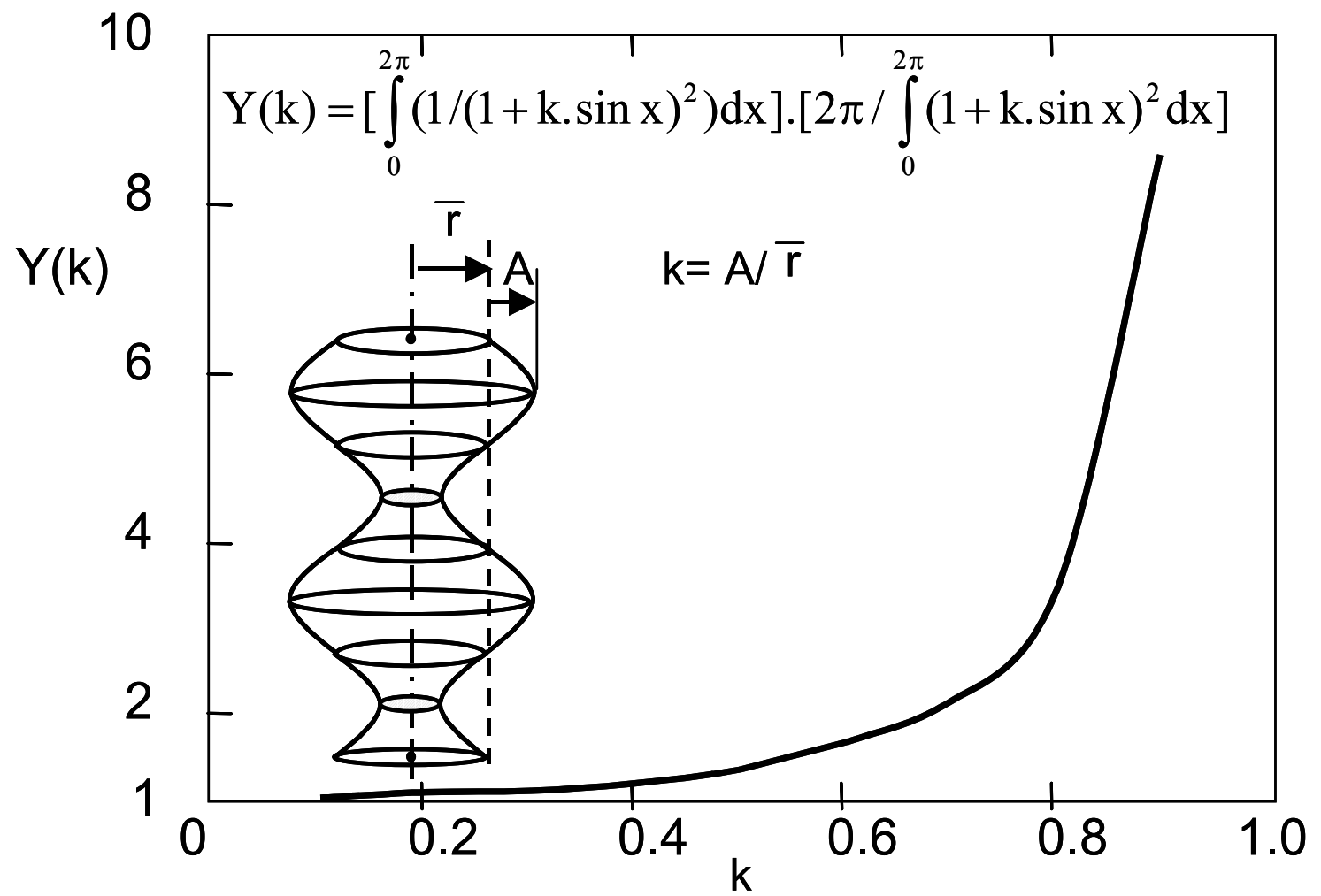

Fig. 5. 


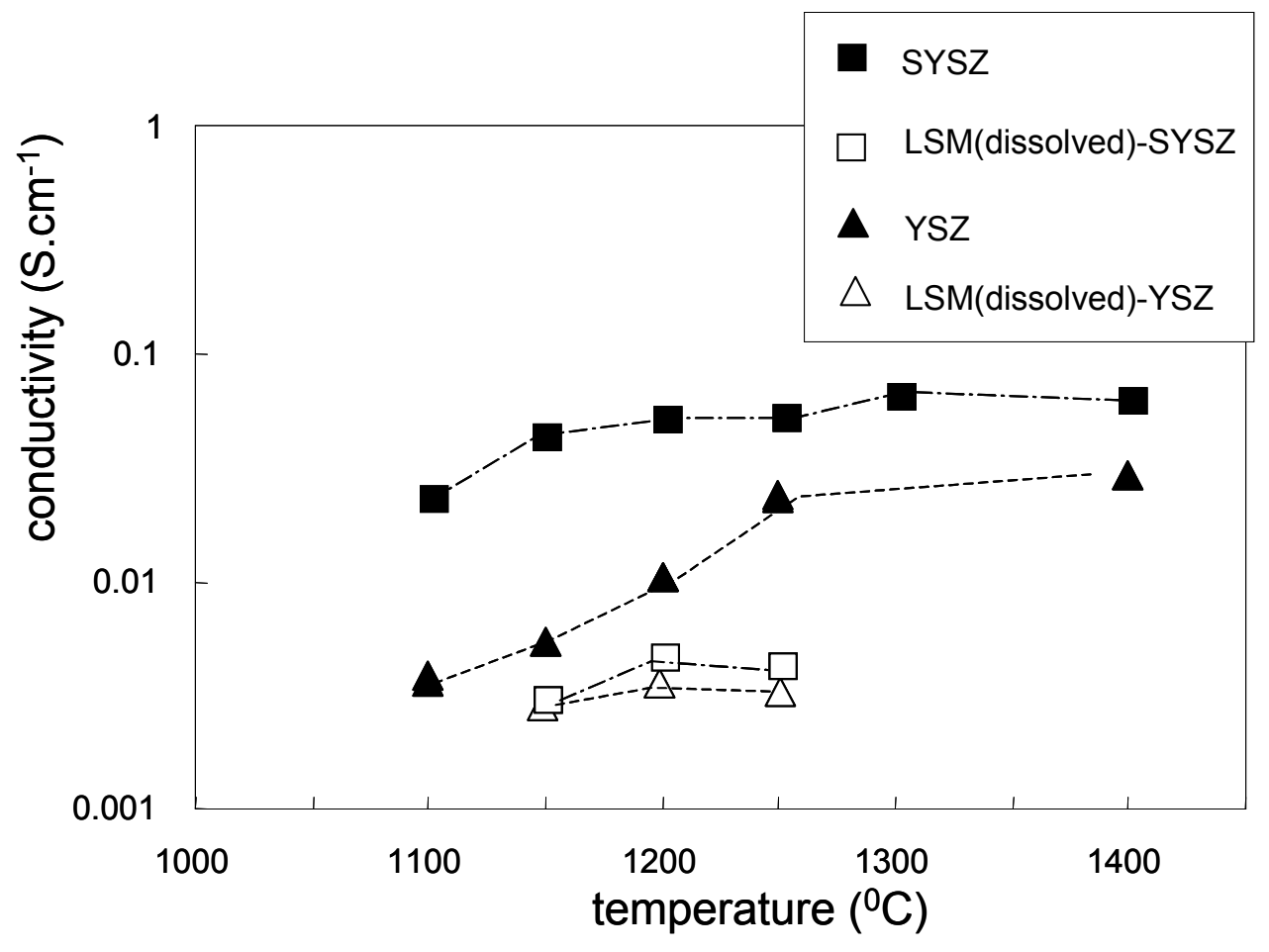

Fig. 6. 


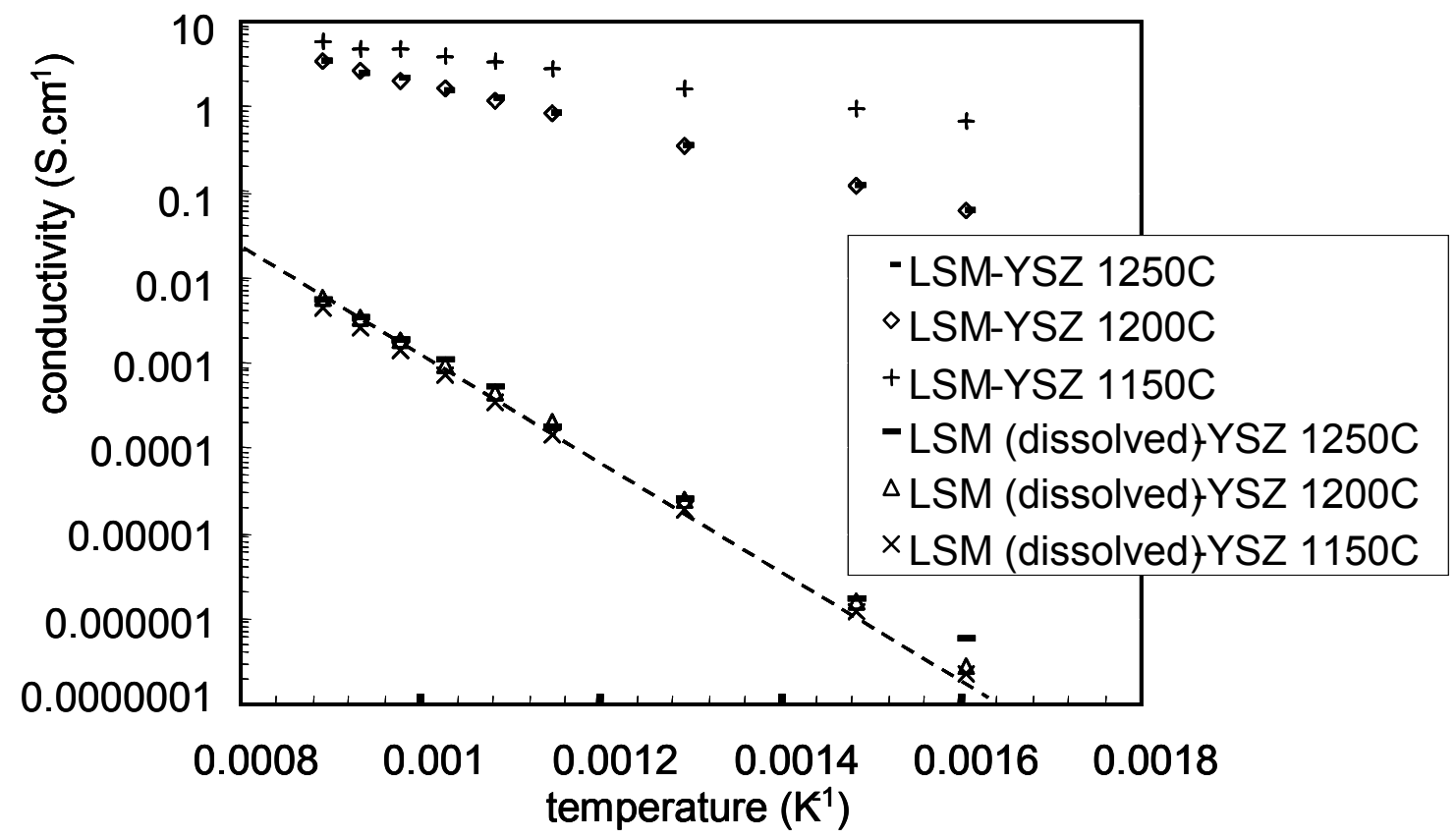

Fig. 7.a

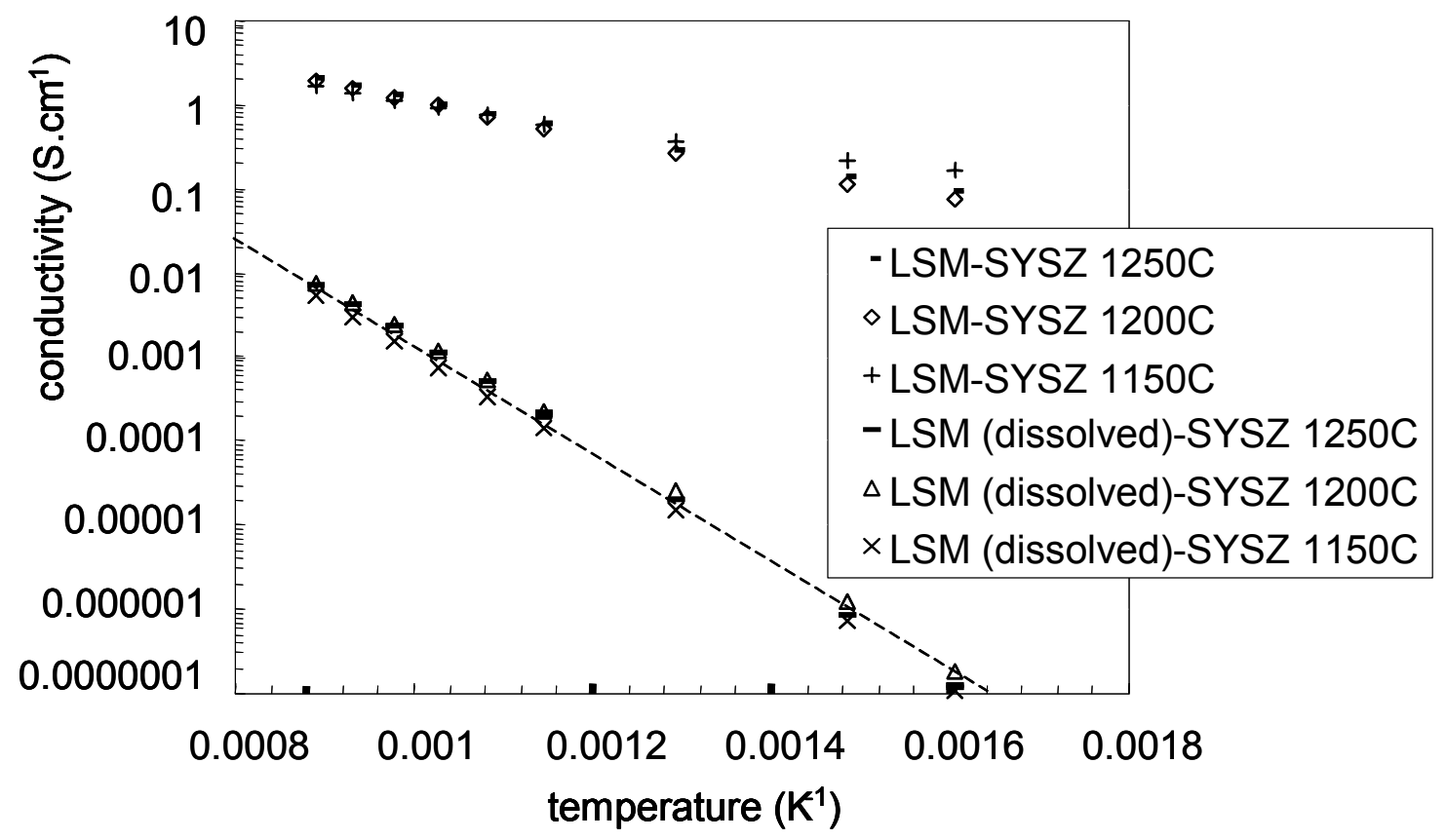

Fig. 7. b 


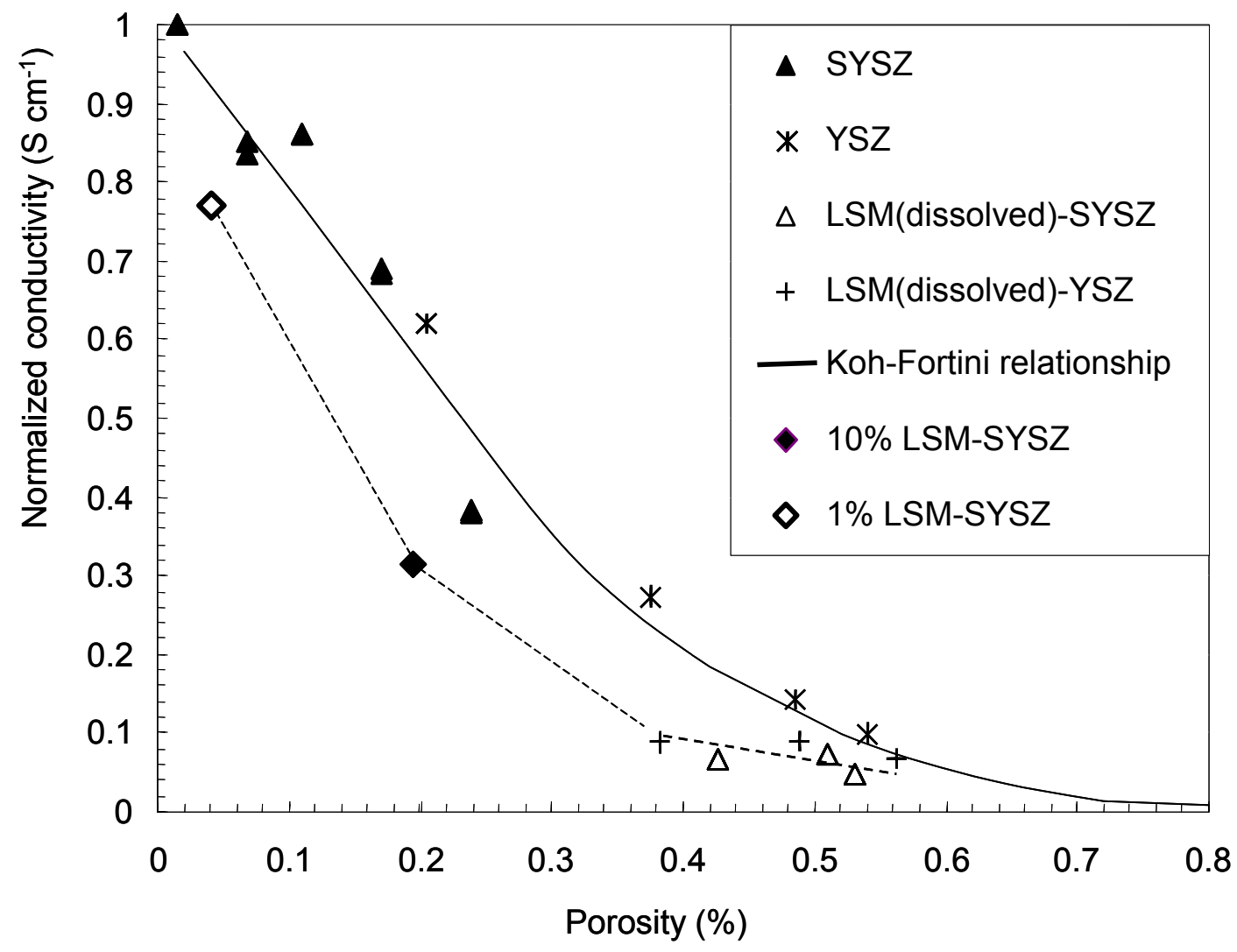

Fig. 8 